\title{
Equation of State for a Quark Gluon Plasma in the Fuzzy Bag Model
}

\author{
R.B. Jacobsen*, C.A.Z. Vasconcellos*, Bardo E.J. Bodmann ${ }^{\dagger}$ and Manfred \\ Dillig**
}

*Instituto de Física, Universidade Federal do Rio Grande do Sul, CEP 91501-970, Porto Alegre,
RS, Brazil
$\begin{array}{r}{ }^{\dagger} \text { Instituto de Física e Matemática, Universidade Federal de Pelotas, CEP 96010-900 Pelotas, Rio } \\ \text { Grande do Sul, Brazil }\end{array}$
${ }^{* *}$ Institut für Theoretische Physik III, der Universität Erlangen-Nürnberg, D91058 Erlangen, Germany

\begin{abstract}
We study two distinct phases of nuclear matter, a baryon-meson phase and a quarkgluon phase (QGP). For the baryon-meson phase we develop an equation of state (EoS) using a quark-meson formulation based on a new version of the fuzzy bag model with scalar-isoscalar, vector-isoscalar and vector-isovector meson-quark couplings and leptonic degrees of freedom as well as the constraints of chemical equilibrium, baryon number and electric charge conservation. For the QGP phase we model an EoS for asymptotically free massless quarks and gluons using the MIT approach and a temperature and baryon chemical potential dependent bag constant, $B(T, \mu)$, which allows an isentropic equilibrium phase transition from a QGP to a hadron gas. Our main results indicate the EoS and static global properties of neutron stars and protoneutron stars at low and moderate values of temperature are slightly modified in comparison to the predictions based on the MIT bag model with a constant $B$.
\end{abstract}

\section{A BAG MODEL WITH A SOFT SURFACE: MODELLING THE MASSES OF NEUTRON STARS}

For the hadron-meson phase, we develop an EoS using a new version of the fuzzy bag model including explicitly the presence of scalar-isoscalar, vector-isoscalar and vectorisovector meson-quark couplings[1, 2, 3, 4]

$$
\begin{aligned}
\mathscr{L}_{F B M} & =\frac{i}{2}\left[\bar{\psi}_{q} \gamma^{\mu} \partial_{\mu} \psi_{q}-\partial_{\mu} \bar{\psi}_{q} \gamma^{\mu} \psi_{q}\right]-B(r) F(r)-\bar{\psi}_{q}\left[m_{q}-g_{\sigma}^{q} \sigma+\left(1+\gamma^{0}\right) V(r)\right] \psi_{q} \\
& +\frac{i}{2}\left[\bar{\psi}_{q} \gamma^{\mu}\left(i g_{\omega}^{q} \omega_{\mu}+i g_{\rho}^{q} \frac{1}{2} \tau \cdot \rho_{\mu}\right) \psi_{q}\right]+\frac{1}{2} \partial_{\mu} \sigma \partial^{\mu} \sigma-\frac{1}{2} m_{\sigma}^{2} \sigma^{2} \\
& -\frac{1}{2} F_{\mu \nu} F^{\mu v}+\frac{1}{2} m_{\omega}^{2} \omega_{\mu} \omega^{\mu}-\frac{1}{4} \mathbf{L}_{\mu \nu} \cdot \mathbf{L}^{\mu \nu}+\frac{1}{2} m_{\rho}^{2} \rho_{\mu} \cdot \rho^{\mu}+\sum_{\lambda} \bar{\psi}_{\lambda}\left[i \gamma_{\mu} \partial^{\mu}-m_{\lambda}\right] \psi_{\lambda}
\end{aligned}
$$

with $F_{\mu v}=\partial_{\mu} \omega_{v}-\partial_{v} \omega_{\mu} ; \mathbf{L}_{\mu v}=\partial_{\mu} \rho_{v}-\partial_{v} \rho_{\mu}$. In these expressions: $\psi_{q}, \sigma, \omega$ and $\rho$ represent, respectively, the quark and meson fields and $g_{\sigma}^{q}, g_{\omega}^{q}, g_{\rho}^{q}$ the quark-meson coupling constants. The main further steps in our calculation are: a) determination of the Euler-Lagrange equations and their solutions for a given confining potential or, equivalently, for chosen suppression functions $F(r)$ and $G(r)$ (with an appropriate 

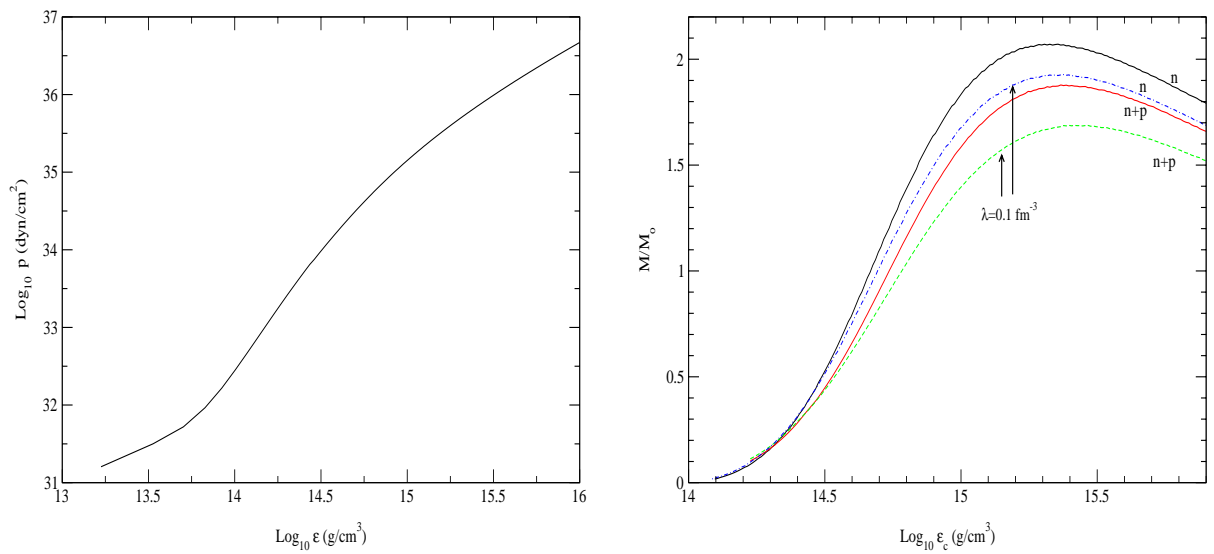

FIGURE 1. Left panel: equation of state as expected in the fuzzy bag model for $\lambda=0.1 \mathrm{fm}^{-3}$ (dashed line) and $\lambda=0.05 \mathrm{fm}^{-3}$ (solid lines). Right panel: maximum mass of neutron star matter as predicted by the fuzzy bag model with $\lambda=0.05 \mathrm{fm}^{-3}$ for different compositions of nuclear matter.

distributional behavior); b) calculation of the energy-momentum tensor in the meanfield approximation[4]; c) determination of the EoS for hadron matter; d) determination of the free parameters of our approach, to reproduce static global properties of nuclear matter; e) integration of the Tolman-Oppenheimer-Volkoff equations to obtain standard plots of global static properties of neutron stars. The figures show our results for the equation of state and for the maximum mass of neutron stars in the fuzzy bag model.

\section{PHASE TRANSITION INVOLVING A HADRON GAS AND THE QUARK GLUON PLASMA}

Thermodynamics tells us that the specific entropy per baryon, $S / B$, in a first order phase transition is smaller in a hadron gas phase than in a QGP phase. On the other hand, the Gibbs criteria for the equilibrium phase across the boundary requires that, at constant temperature, pressure and chemical potential of both hadron and QGP phases are related through $P_{Q G P}=P_{H} ; \mu_{Q G P}=\mu_{H}$, for a conserved baryon current given as $N_{Q G P} / 3+N_{H}=$ constant. Thus, Gibbs criteria yields at constant temperature $S /\left.B\right|_{Q G P}>S /\left.B\right|_{H}$ i.e., a phase transition for which the specific entropy per baryon, $S / B$, is discontinuous across the phase boundary. To recover continuity as well as entropy and baryon number conservation, we follow the formalism developed by A. Leodinov et 
al[5], introducing a temperature and baryon chemical potential dependence on the bag pressure $\frac{S_{Q G P}-\frac{\partial B(\mu, T)}{\partial T}}{N_{Q G P}-\frac{\partial B(\mu, T)}{\partial T}}=\frac{S_{H}}{N_{H}}$, which yields, after extending the original formalism to consider mesonic interactions, up to $\mathscr{O}\left(T^{4}\right)$

$$
B(\mu, T)=B_{0}+\frac{37}{90} \pi^{2} T^{4}+\left(\frac{\mu^{2}}{9}-\left(\frac{\mu}{\theta}\right)^{2} \frac{\mu^{2}}{81}\right) T^{2}-\alpha_{S}(\mu, T)\left[\frac{11}{9} \pi T^{4}+\frac{2}{9 \pi} \mu^{2} T^{2}+\frac{\mu^{4}}{81 \mu^{3}}\right]
$$

for the QCD running coupling constant $\alpha_{S}(\mu, T)$ a slowly varying function of $T$ at $\theta \equiv\left(\mu^{2}-m^{2}\right)^{1 / 2}$. In modelling the EoS for the QGP, we assume asymptotically free massless quarks and gluons and the MIT approach. Thus, the energy density of the gas is given as

$$
\varepsilon=\frac{E}{V}=B(\mu, T)+\frac{\gamma_{Q}}{(2 \pi)^{3}} \int k d^{3} k\left(n_{k}(T)+\bar{n}_{k}(T)\right)+\frac{\gamma_{G}}{(2 \pi)^{3}} \int \frac{k d^{3} k}{e^{\beta k}-1}
$$

while the pressure is

$$
p=-B(\mu, T)+\frac{1}{3}\left\{\frac{\gamma_{Q}}{(2 \pi)^{3}} \int k d^{3} k\left(n_{k}(T)+\bar{n}_{k}(T)\right)+\frac{\gamma_{G}}{(2 \pi)^{3}} \int \frac{k d^{3} k}{e^{\beta k}-1}\right\}
$$

with the baryon density $\rho_{B}=\frac{1}{3} \frac{\gamma_{Q}}{(2 \pi)^{3}} \int k d^{3} k\left(n_{k}(T)-\bar{n}_{k}(T)\right)$. The statistical factors for degeneracy factors of quarks and gluons are $\gamma_{Q}=12$ and $\gamma_{G}=16$, respectively. Above the thermal Fermi distribution functions are defined, for quarks carrying baryon number $1 / 3$, as $n_{k}(T)=\frac{1}{e^{\beta(k-\mu / 3)}+1} ; \bar{n}_{k}(T)=\frac{1}{e^{\beta(k+\mu / 3)}+1}$.

\section{RESULTS AND CONCLUSIONS}

Our main results indicate the EoS and static global properties of neutron stars and a PQG star at low and moderate values of temperature, as the ones found in protoneutron stars, with the inclusion in the theoretical treatment of a temperature and baryon chemical potential dependence on the bag pressure, are slightly modified in comparison to the predictions based on the MIT bag model with a constant $B$.

\section{REFERENCES}

1. $\quad$ F.G. Pilotto, C.A.Z. Vasconcellos and H.T. Coelho, Mod. Phys. Lett. A17 (2002) 543.

2. $\quad$ F.G. Pilotto, The European Physical Journal C28 (2003) 535.

3. C.A.Z. Vasconcellos, H.T. Coelho, F.G. Pilotto, B.E.J. Bodmann, M. Dillig and M. Razeira, Eur. Phys. J. C4 (1998) 115. Mod. Phys. Lett. A17 (2002) 543.

4. C.A.Z. Vasconcellos and M. Dillig, in Proceedings of the International Workshop on Strong Magnetic Fields and Neutron Stars, Cuba, 2003 (to be published).

5. A. Leodinov et al., Phys. Rev. D50 (1994) 4657. 
Copyright of AIP Conference Proceedings is the property of American Institute of Physics and its content may not be copied or emailed to multiple sites or posted to a listserv without the copyright holder's express written permission. However, users may print, download, or email articles for individual use. 